\title{
An inexpensive method of slit-lamp photography
}

\author{
V. T. THALLER \\ From St Thomas's Hospital, Lambeth Palace Road, London SE1 7EH
}

SUMmARY A method for slit-lamp photography is described with a conventional Haag-Streit slit-lamp microscope in conjunction with a $35 \mathrm{~mm}$ single lens reflex camera with a $50 \mathrm{~mm}$ standard lens. Coupling of the camera to the slit-lamp is simply and reversibly achieved with the use of a specially designed adapter ring and requires no modification to either camera or slit-lamp. Details of the adapter are given.

Ophthalmologists sometimes wish to take anterior segment photographs for record or teaching purposes. This is not always easy, either because the expensive specialised equipment is not at hand or because its use is not straightforward and reliable.

The method here described for taking such photographs is a simple and inexpensive alternative yielding surprisingly good results. It is available to any interested ophthalmologist who possesses a 35 $\mathrm{mm}$ single lens reflex (SLR) camera and has access to a slit-lamp.

\section{Principle}

A correctly focused slit-lamp emits parallel light rays from its eye piece which would theoretically form an image at infinity. A camera with its lens focused on infinity will accept parallel light rays and form them into a focused image on the film plane (in the case of a single lens reflex on the focusing screen when not actually taking a photograph). If the slit-lamp and camera are aligned so that their optical axes coincide, an image of the eye under examination is formed in the camera.

\section{Adapter ring}

The above principle holds for any slit-lamp. The details which follow apply to the Haag-Streit $\times 10$ eyepiece, which has a very convenient collar and flange for the purpose intended.

A machined plastic (or other convenient material) ring is manufactured to the dimensions in Fig. 1. To Correspondence to Dr V. T. Thaller, Moorfields Eye Hospital, City Road, London EC1V 2PD. ensure a snug 'push' fit of the adapter on to the microscope eyepiece a Neoprene 'O' ring (Fig. 1, G) is used in the central adapter orifice. The adapter is eased on to the eye piece as far as it will go before being stopped by the flange. The eyepiece barrel can then be replaced in its tube on the microscope and pushed home without the adapter affecting the eyepiece position.

To the aforementioned adapter a readily available filter thread ring (commercially available from photographic retailers to fit filter holders such as the Cokin system) is permanently attached by 4 screws. The thread diameter is chosen to fit the camera lens being used (most commonly $49 \mathrm{~mm}$ diameter for the standard lens). The camera can then be mounted on the slit-lamp as shown in Fig. 2.

The adapter ring as described above can easily be manufactured in most hospital engineering workshops at minimal cost.

\section{Focusing}

Focusing is done in the normal slit-lamp fashion by forward/backward movement of the whole instrument while viewing through the camera eyepiece.

In SLR cameras with a matt (ground glass) focusing screen the viewfinder image may appear rather dim and grainy. This in no way affects the picture exposure or the quality but may impede easy focusing. If such a camera has interchangeable focusing screens it is well worth investing in a clear screen with cross hairs for focusing, as this will afford near normal viewing brightness and clarity.

The instrument is in focus when both the cross hairs and subject image are simultaneously sharp. For this 


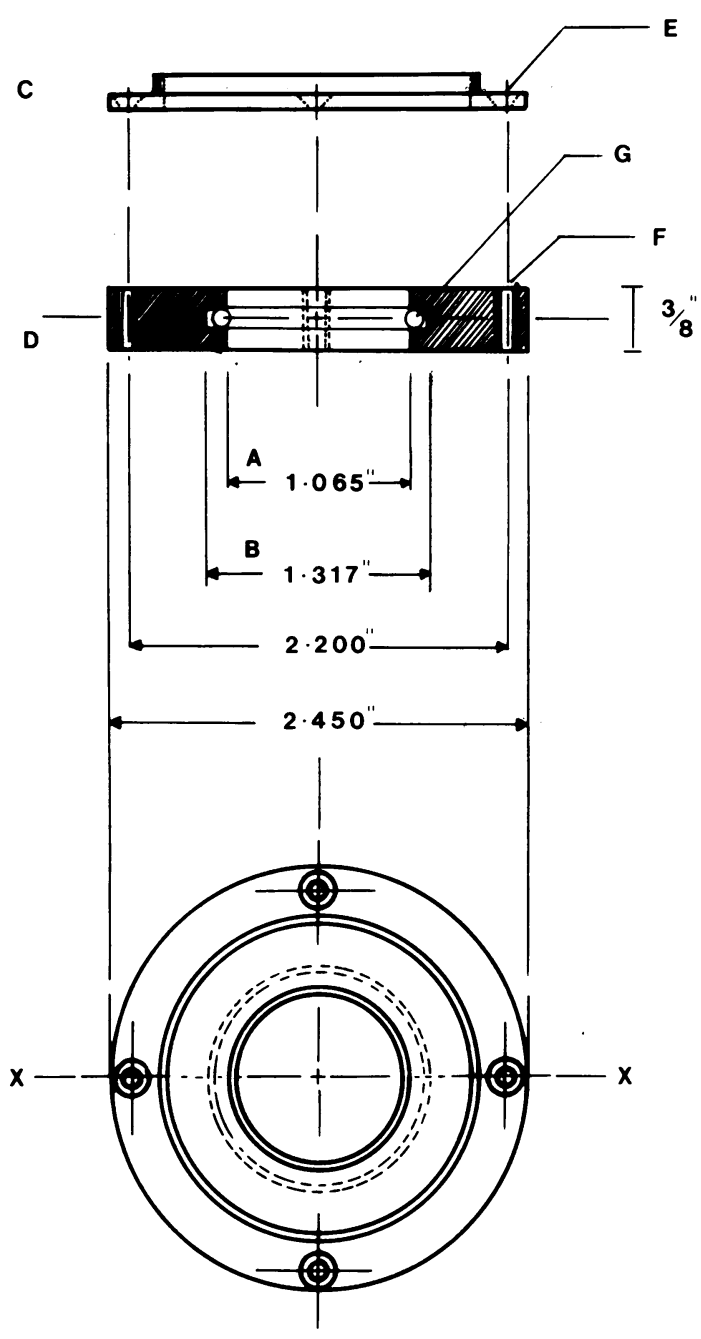

Fig. 1 Adapter detail (measurements in inches; $\times 25 \cdot 4=\mathrm{mm}$ ). A: Eyepiece outside diameter (push fit). B: O-ring groove diameter (to allow expansion). C: Filter thread adapter ring (commercially available). D: Eyepiece adapter ring section on $X-X$ (material: $3 / 8$ inch Darvic grey). E: 6 BA counter-sunk (flush). F: $4 \times 6$ BA 3/8 inch tapped holes (on 2.200 inches centres). G: O-ring (0.215 inch) kit UC 2901, BS 1806.

adjustment the eye piece dioptric correction should be set to zero (the photographer should wear his distance correction if any).

\section{Exposure}

Correct exposure is important if good quality photographs are to be obtained. This will vary according to the subject and type of photograph required. A rough guide is given in Table 1 . Some experimentation will be required for optimal results.

Exposure control is exercised in 3 ways: (1) Subject illumination can be adjusted by setting the slit-lamp voltage, slit width, and filtration. For most purposes a wide slit at 5 volts is suitable. Very narrow slit beams do not show up well in clear media. When using a narrow slit it is sometimes desirable to have simultaneous background illumination. This may be supplied by an anglepoise desk lamp or photographic flash-gun placed at an appropriate distance (found by experiment). (2) A shutter speed appropriate to the subject and illumination must be chosen (see Table 1). Provided the subject is co-operative and able to keep still, shutter speeds as slow as $1 / 8$ th $s$ can give sharp photographs. (3) A moderately sensitive (fast, high ASA rating) film is required to avoid the necessity for long exposures, thus minimising the risk of blurring from inadvertent subject movement.

As illumination is from a tungsten light source a tungsten balanced colour film is recommended. An appropriate colour compensating filter (Wratten 80B) can be used on the camera lens if a daylight balanced colour film is used. The exposure guide in Table 1 is based on the use of Ektachrome 160 ASA tungsten colour slide film.

It should be noted that exposure control cannot be achieved by altering the camera lens aperture setting (this should be fully open, cf. Table 2). This is because the aperture stop in this optical combination is set in the slit-lamp microscope. Closing the camera lens aperture merely acts as a field stop, cutting down the field size of the image without usefully altering its luminosity. This effect becomes noticeable at aperture settings smaller than $\mathrm{f} 4$ in the set-up here described.

\section{Discussion}

The exposure settings suggested in Table 1 are intended as a starting guide and may require modification in the light of personal experience. It will be noted from Table 1 that the shutter speeds are specified: It is recommended that automatic cameras are used in the manual mode. More consistent results will then be obtained, as the automatic exposure mechanism is easily misled by the uneven lighting conditions and reflections encountered in this type of work.

Image magnification is affected by both the slitlamp microscope setting and by the camera lens focal length. In the case of the Haag-Streit no exposure compensation is required when switching from the $\times 1$ to $\times 1.6$ objective magnification. If a camera lens of greater focal length than the standard $50 \mathrm{~mm}$ is used (e.g., medium telephoto $135 \mathrm{~mm}$ ) a considerable image magnification is obtained. In practice the extra 

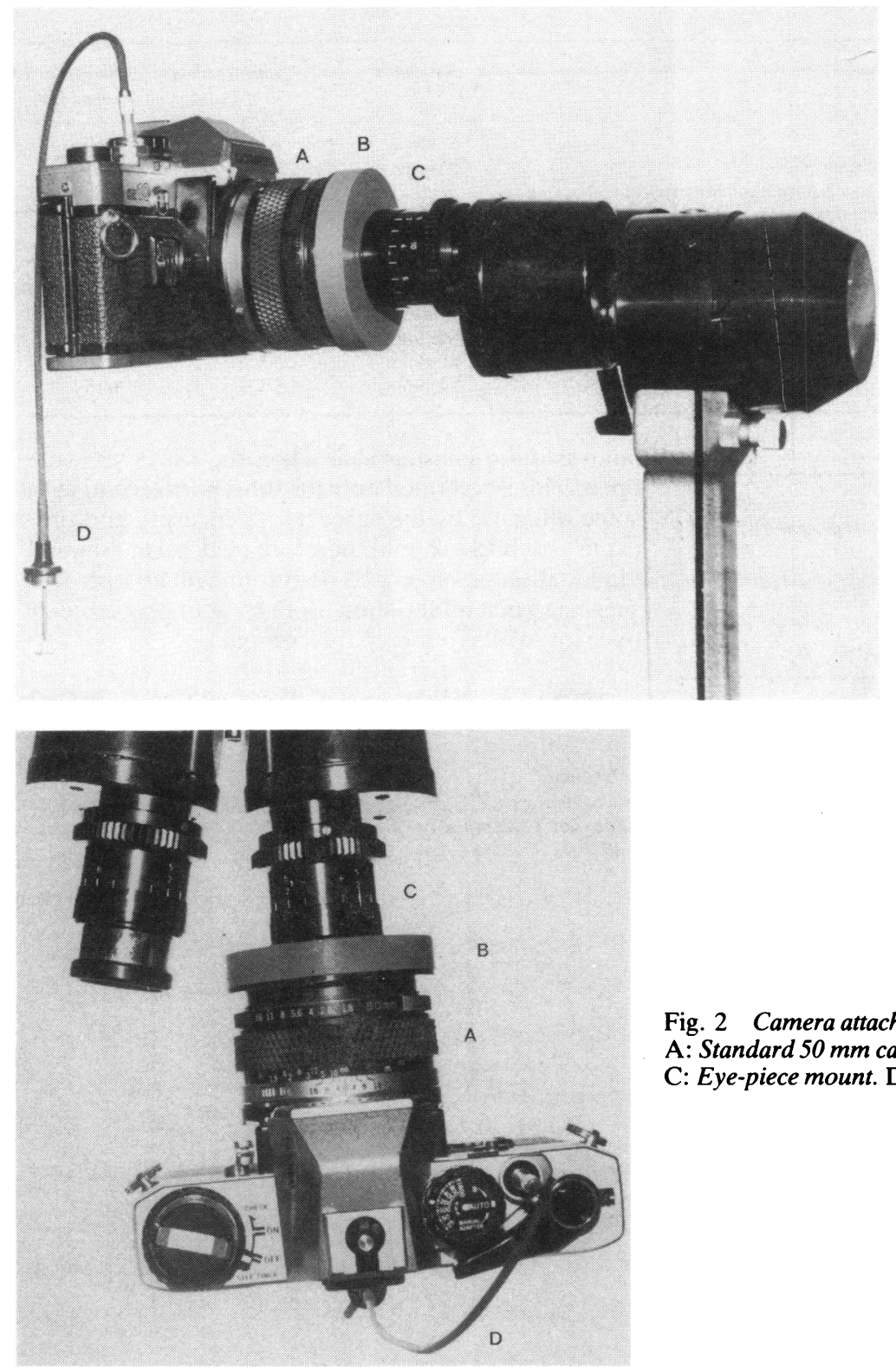

Fig. 2 Camera attached to slit-lamp by adapter ring. A: Standard $50 \mathrm{~mm}$ camera lens. B: Adapter ring. C: Eye-piece mount. D: Cable release.

magnification is seldom required and can be gained only at some expense to image definition. It must also be remembered that the extra leverage and weight of a longer lens greatly increases the loading on the adapter and slit-lamp microscope and this may prove critical if no additional camera support is used. The loading of an average SLR camera with standard lens is readily withstood by the slit-lamp microscope mechanism and is in no way detrimental to it.
To reduce the possibility of camera shake and defocusing it is recommended that a cable shutter release is used (Fig. 2D).

It is of course practicable to take fundus photographs with the equipment described using a corneal contact lens or a Hruby lens. Goniophotography is also possible through a goniolens.

For convenience a pre-exposure check-list is given in Table 2. 
Table 1 Exposure guide

\begin{tabular}{|c|c|c|c|c|}
\hline Subject & Note & Slit width & Lamp voltage & $\begin{array}{l}\text { Shutter } \\
\text { speed (s) }\end{array}$ \\
\hline Survey/conjunctiva & & Wide & $6 \mathrm{~V}$ & $1 / 60$ \\
\hline Cornea: optic section & Oblique illumination & Narrow & $7 \cdot 5 \mathrm{~V}$ & $1 / 15$ \\
\hline surface & \pm Staining with fluorescein or rose Bengal & Wide & $5 \mathrm{~V}$ & $1 / 30$ \\
\hline fluorescein & Cobalt blue filter & Wide & $7 \cdot 5 \mathrm{~V}$ & $1 / 15$ \\
\hline Iris & & Medium/wide & $5-7 \cdot 5 \mathrm{~V}$ & $1 / 30$ \\
\hline Chamber angle & Gonioscopic contact lens & Medium & $5 \mathrm{~V}$ & $1 / 30-1 / 60$ \\
\hline Lens: optic section & Oblique illumination & Narrow & $7 \cdot 5 \mathrm{~V}$ & $1 / 8-1 / 15$ \\
\hline direct illumination & & Wide & $7 \cdot 5 \mathrm{~V}$ & $1 / 30$ \\
\hline retroillumination & Frontal illumination from laterally displaced slit & Medium & $7 \cdot 5 \mathrm{~V}$ & $1 / 15$ \\
\hline Fundus: disc/post. pole & Contact lens & Medium & $5 \mathrm{~V}$ & $1 / 30$ \\
\hline periphery & 3-Mirror contact lens & Medium & $5 \mathrm{~V}$ & $1 / 15$ \\
\hline
\end{tabular}

Table 2 Check list

\begin{tabular}{|c|c|c|}
\hline Slit-lamp: & $\begin{array}{l}\text { eye piece } \\
\text { lamp voltage } \\
\text { slit width } \\
\text { magnification } \\
\text { focus } \\
\text { aperture } \\
\text { shutter speed }\end{array}$ & $\begin{array}{l}\text { set correction to zero } \\
\text { see exposure guide (Table } 1 \text { ) } \\
\text { as required } \\
\times 1 \text { or } \times 1 \cdot 6 \text { as required } \\
\text { on infinity }(\infty) \\
\text { set maximum (e.g., } 1.8) \\
\text { see exposure guide (Table } 1 \text { ) }\end{array}$ \\
\hline
\end{tabular}

A concise treatment of slit-lamp photography and its problems is available. ${ }^{1}$

Finally, a note for the patient's, rather than the operator's, benefit. It must be remembered that the heat and light intensity generated by a standard slit- lamp is quite considerable when the slit is set wide open. This is tolerated only for short periods and with some difficulty by the subject. All focusing and preexposure checks should therefore be done at minimal illumination or subject co-operation will be lost. It is suggested that all slit-lamp users try acting as subjects once in a while to top up their empathy.

I am grateful to the Department of Bioengineering, St. Thomas's Hospital for the construction of the prototype adapter.

\section{Reference}

1 Meyner EM. Atlas of Slit Lamp Photography. Stuttgart: Enke, 1976. 